ISBN 978-93-84422-76-9

6th International Conference on Developments in Engineering and Technology

(ICDET-2017)

Bangkok (Thailand) Feb.6-7, 2017

\title{
In Situ-Preparation and Characterization of Silver-Acrylic Hydrogel Matrix Nanocomposites: It's Drug Release Property
}

\author{
Himadri Sekhar Samanta ${ }^{1}$, Chiranjib Bhattacharjee ${ }^{1}$ \\ ${ }^{1}$ Department of Chemical Engineering, Jadavpur University, Kolkata - 700032, India
}

\begin{abstract}
Hydrogels are water-insoluble crosslinked hydrophilic networks capable of retaining a large amount of water. The present work aimed to develop a novel carboxy-methyl celluloselacrylamide based hydrogel which could behave both as a nanoreactor and an immobilizing matrix for silver nanoparticles (AgNPs) with promising drug delivery vehicle.The hydrogel containing AgNPs were prepared free radical polymerization using varying amounts of the crosslinker, silver nitrate, initiator etc. followed by in situ reduction with plant leaf extract, acted as both reducing agent and stabilizing agent, was added to the reaction medium. Analysis and characterization of the so obtained both hydrogels were performed through monitoring swelling behavior, FTIR spectroscopy, SEM \& EDX, UV-vis spectrophotometer, XRD and TEM. pH response of this only hydrogel \& nanocomposite hydrogel shows drug release behaviour in different $\mathrm{pH}(1.5 \& 7.5)$ which is made it suitable for drug delivery applications.
\end{abstract}

Keywords: Green synthesis, silver-nanoparticles, hydrogels, Characterization, drug delivery

\section{Introduction}

Hydrogels have three-dimensional polymeric networks that are fabricated from polymers stabilized through physical or chemical crosslinking. They absorb large quantities of water without losing their structural integrity [1]. Due to the presence of water solubilizing groups, such as $-\mathrm{OH},-\mathrm{COOH},-\mathrm{CONH}_{2},-\mathrm{CONH}-$, and $-\mathrm{SO}_{3} \mathrm{H}$, these hydrogels show higher hydrophilicity. Their swollen state results from a balance between the dispersing forces acting on hydrated chains and cohesive forces that do not prevent the penetration of water through the network [2]. Based on these properties the hydrogels have been used recently as templates for production of metallic nanoparticles e.g silver, gold etc.. due to their ease of preparation, good biocompatibility, and relatively large surface area So, hydrogel nanocomposites, which includes the incorporation of inorganic -nanoparticles inside 3D polymeric networks, have been attracted great interests in recent years because of their intrinsic advantages over pure hydrogels or inorganic nanoparticles and remarkably improvement some properties of hydrogel such as mechanical toughness, large deformability, high swelling/deswelling rates, excellent electrical conductivity, antimicrobical effects, and optical properties, and high transparency [3-6]. To improve the dispersion of nanoparticles inside the hydrogel matrix, and also partially prevent the formation of aggregates, the 
entrapment of silver cations by hydrogel matrix followed by reduction with common reducing agents has been preferred to the simple mixing of the two components and the polymerization in the presence of presynthesized silver nanoparticles[7-9].

Mangifera indica well as known medicinal plant in folklore for its medicinal properties due to contains several flavonoids, alkyl esters, terpenes, sterols, fatty acids, and polysaccharides. In herbal medicine the seeds of it are mainly used as tonic, astringent, diuretic, and are also recommended in asthma, bronchitis, urinary discharges, hiccoughs, ozoena, heart trouble and other diseases of brain.

In this work, to achieve novel and better antibacterial products, semi IPN-hydrogel-silver nanoparticle composites (SNA) are developed in the present investigation \& introduce an easy way to synthesis a silver without using any extra reducing agent at room temperature. This system will make its reach to the site of action through GI tract and will show the $\mathrm{pH}$ responsive behavior and will release the Ciprofloxacin $(\mathrm{CFx}$ in

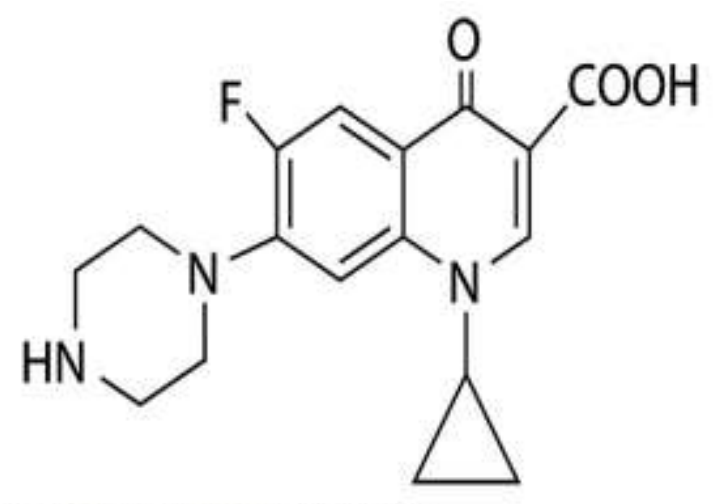

FIG. 1 Structure of ciprofloxacin drug

\section{Experimental}

\subsection{Materials}

Acrylamide(AAm), $N, N^{\prime}$ 'bimethylene acrylamide(BMA) and ammonium persulphate were of chemical pure grade. Ciprofloxacin was obtained from Ranbaxy Pvt. Ltd., India. All chemicals were used without further purification. Double-distilled(DD) water is used throughout the experiment.

\subsection{Preparation of hydrogel}

In general, Mango leaf extract was added to DDW at a three-neck reactor with stirring (200 rpm). After homogenization, different concentrations of $\mathrm{AgNO}_{3}(0.0025,0.005,0.01$ and $0.02 \mathrm{M})$ was added to the reaction mixture and was stirred for further $30 \mathrm{~min}$. The dissolved oxygen of the reaction mixtures was removed by purging nitrogen gas in the reaction mixtures before addition of CMC, acrylamide, MBA and required amounts of redox pair of initiator i.e. ammonium persulfate and sodium metabisulfite and the reaction was then continued at this temperature till the reaction mixtures gelled. For preparation of the without silver composite gel, the required amounts CMC, AAm, MBA was dispersed in the aqueous solution followed by a similar polymerization in this aqueous dispersion. The gel obtained was disintegrated in a blender, washed with water and then isopropyl alcohol, followed by filtration and finally dried to constant weight at $30{ }^{\circ} \mathrm{C}$ in a vacuum oven. 


\subsection{Characterization of the hydrogel}

Preliminary characterization of the hydrogels containing silver nano-particles was carried out using UV-vis spectrophotometer with a scan range of 300-600 nm, Fourier transform infrared spectra (FTIR), wide angle $\mathrm{x}$-ray diffraction (XRD), morphology of the gold coated gel samples were observed by the scanning electron microscope (SEM) and Transmission electron microscopy (TEM) Transmission electron microscopy (TEM) was used to determine the size of silver nano-particles inside the hydrogel..

\subsection{Study of equilibrium swelling and Swelling kinetics}

For the study of the dynamic swelling properties of the hydrogels, the dried hydrogel sample was weighed $\left(\mathrm{m}_{\mathrm{d}}\right)$ and immersed in the distilled water. The swollen hydrogels were withdrawn from the solution at different time intervals $(t)$ and weighed $\left(\mathrm{m}_{\mathrm{t}}\right)$ after removing the excess surface water with a filter paper. The swelling experiments were continued till the hydrogels reached its equilibrium swelling value $\left(\mathrm{m}_{\mathrm{e}}\right)$. The swelling ratio $\left(\mathrm{S}_{\mathrm{t}}\right)$ and the equilibrium swelling ratio $\left(\mathrm{S}_{\mathrm{e}}\right)$ and kinetics was determined as

$$
S_{\mathrm{t}}(g / g)=\frac{\mathrm{m}_{\mathrm{t}}-\mathrm{m}_{\mathrm{d}}}{\mathrm{m}_{\mathrm{d}}} \quad \text { (1a) } S_{\mathrm{t}}=\frac{\mathrm{W}_{\mathrm{e}}^{2} \mathrm{k}_{\mathrm{S} 2} \mathrm{t}}{1+\mathrm{k}_{\mathrm{S} 2} \mathrm{~W}_{\mathrm{e}} \mathrm{t}}=\frac{\mathrm{r}_{0} \mathrm{t}}{1+\mathrm{k}_{\mathrm{S} 2} \mathrm{~W}_{\mathrm{e}} \mathrm{t}}
$$

Here, $\mathrm{k}_{\mathrm{s} 2}$ is rate constant and $\mathrm{r}_{0}$ is initial rate of swelling.

\subsection{Study of the drug loading and Drug release of polymer matrix}

For loading of the ciprofloxacin in the hydrogel samples, the gel samples were allowed to swell in water containing the drug of the known concentration for $48 \mathrm{~h}$ at $37^{\circ} \mathrm{C}$. The wet samples were then removed from the solution and dried. The drug loading (DL) and the entrapment efficiency of the sample was determined as

$$
\mathrm{DL}(\mathrm{mg} / \mathrm{g} \text { hy drogel sample })=\frac{\mathrm{m}_{\mathrm{d}}-\mathrm{m}_{\mathrm{i}}}{\mathrm{m}_{\mathrm{i}}} \quad \text { (4) Entrapment efficiency }(\%)=\frac{\mathrm{m}_{\mathrm{d}}-\mathrm{m}_{\mathrm{i}}}{\mathrm{m}_{\mathrm{o}}} x 100
$$

Where $m_{d}$ is the weight of the dried drug loaded hydrogel sample, $m_{i}$ is the weight of the hydrogel sample and $\mathrm{m}_{\mathrm{o}}$ is the weight of the drug dissolved in water.

In vitro drug release of the ciprofloxacin from the hydrogel samples was carried out at $37{ }^{\circ} \mathrm{C}$ at $50 \mathrm{rpm}$ in $100 \mathrm{ml}$ of buffer solution ( $\mathrm{pH} 1.5$ and 7.5) for 25-30 h. The concentration of the drug in the withdrawn solution was analyzed by a UV-Vis spectrophotometer at $274 \mathrm{~nm}$ for ciprofloxacin using a calibration curve constructed from a series of ciprofloxacin solutions of known concentrations. The drug release $\%$ was obtained as

$$
\text { Drug release } \%=\frac{\mathrm{m}_{\mathrm{d}}-\mathrm{m}_{\mathrm{r}}}{\mathrm{m}_{\mathrm{r}}} \times 100
$$

Where $\mathrm{m}_{\mathrm{r}}$ is the amount of the drug released in the solution and $\mathrm{m}_{\mathrm{d}}$ is the weight of the drug loaded dry gel sample. 


\section{Results and discussion}

\subsection{Characterization of the hydrogel}

A typical absorption spectrum of the silver colloidal solution is shown in Fig.2ai, 2aii \& 2aiii. This band is assigned to the surface plasmon absorption (SPR) of the nanosilver particles. It peaks at $418 \mathrm{~nm}$ and has a band width at half maximum of $130 \mathrm{~nm}$, which is an indication of the particle size distribution [10]. Fig.2ai shows the UV-vis spectra of the Ag nano-particles in the hydrogel but Fig.2aii don't show any characteristics peaks due to absence of nanoparticle.

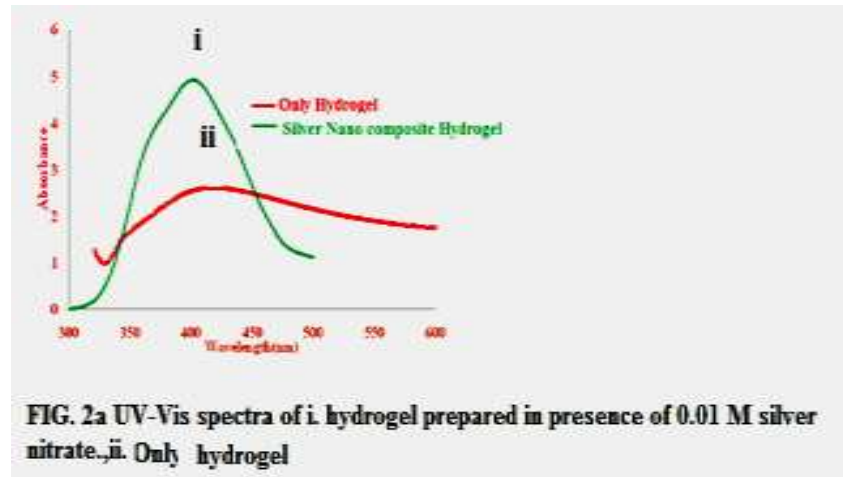

This analysis confirms the formation of highly dispersed $\mathrm{Ag}^{0}$ nanoparticles in the copolymeric hydrogels. SEM of hydrogel (SNA0) \& SNA were investigated \& shown in Fig.2bi \& ii. It is observed from SEM of SIPN (Fig.2bi) that CMC and polyacrylamide shows good compatibility in SIPN. Further, introduction of mango extract facilitates surface and network structure of the IPN gel which is desirable for good absorption properties whereas Ag NPs-loaded hydrogel exhibits smaller nanoparticles distributed throughout the hydrogel networks so rough morphologies results in Fig.2bii. In both Fig.2ci EDX quantitative analysis confirms the nanostructure which contains about $67.54 \mathrm{wt} \%$ carbon, and about $32.07 \mathrm{wt} \%$ oxygen, while Fig.2cii shows the elemental content as follows: oxygen, $37.86 \mathrm{wt} \%$; carbon, $55.63 \mathrm{wt} \%$; $\mathrm{Ag}, 6.51 \mathrm{wt} \%$ would reveal that, higher content of Ag nano-particles is obtained when the hydrogel containing silver nano-particles. In Fig.2di \& ii crystallinity of the only hydrogel (SNA0) and semi-IPN hydrogel-silver nanocomposite (SNA) was confirmed by the analysis of X-ray diffraction (XRD) Pattern, resectively. The dry powders of hydrogel SNA0 and SNA were used for XRD analysis. In Fig.2cii the four diffraction peaks were obtained at an angle, $2 \theta=38.69,49.53,64.50$, and 77.6 which could be attributed to the Brags reflections of (111), (200), (220), and (311) planes of face centred cubic (FCC) structure of Ag NPs but this types of peak was not observed in the case of hydrogel in Fig.2ci. This analysis confirms the formation of highly dispersed $\mathrm{Ag}^{0}$ nanoparticles in the hydrogels. 

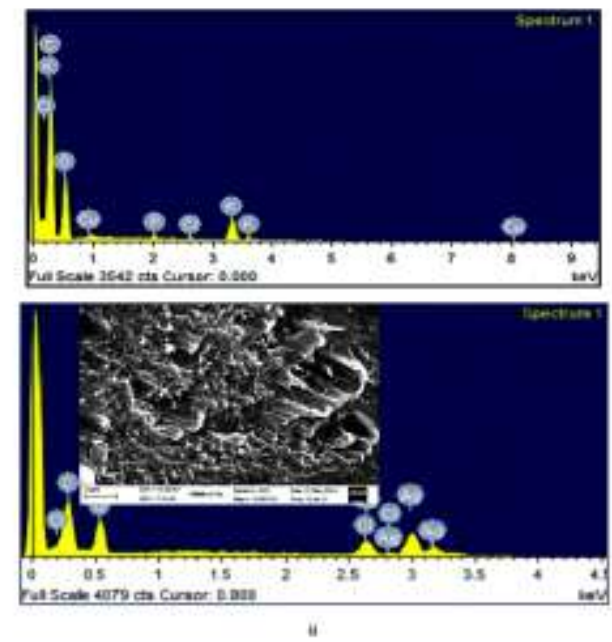

FIG. 2 e SEM(iaset) \& EDAX inage of i. semi-IPN hydrogels (SNi0) \& il. silver composite hydrogel(SNA3)

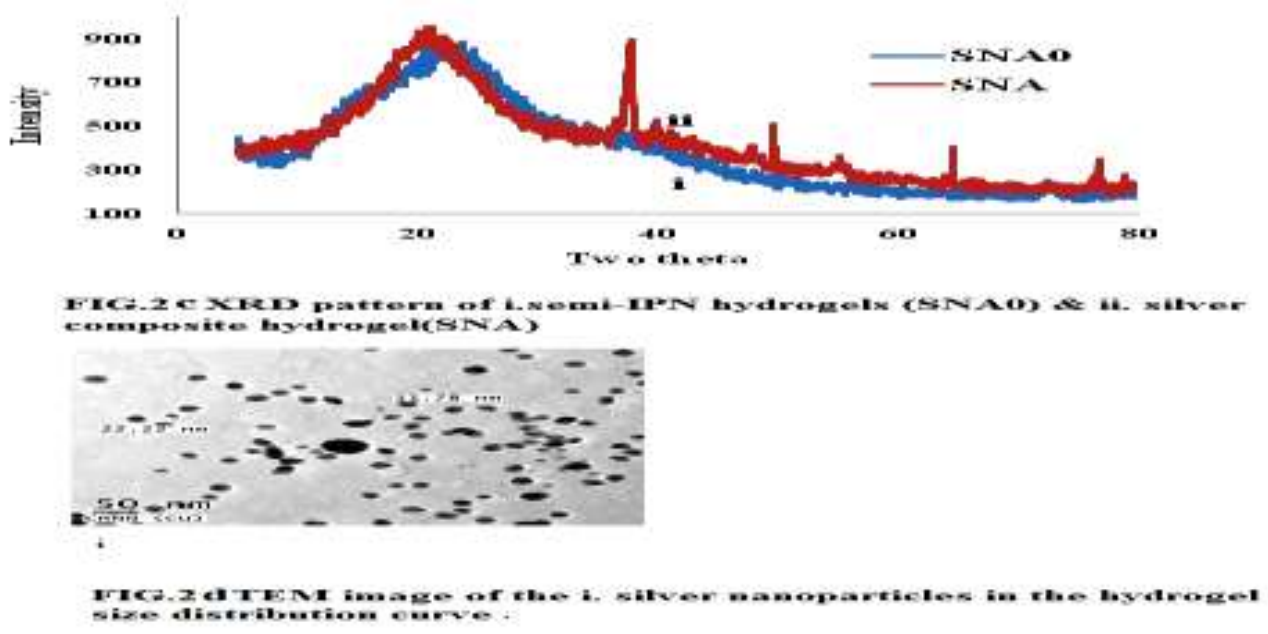

In Fig.2d TEM images was utilized for more precise study of the presence of silver nanoparticles in the SNA hydrogel structure. In Fig.2di TEM image showed a highly uniform distribution of silver nanoparticles in the biopolymer matrix. It was confirmed that the silver nanoparticles in the cross-linked networks were spherical and highly dispersed in the biopolymer matrix with an approximate size of 10-20 nm. It is also obvious that, the nano-particles do not form aggregates. This may be due to excellent stabilization of silver nano-particles by carboxylate anions present in the gel macromolecular chain.

\subsection{Study of swelling properties of the hydrogels and In vitro drug release study}

Drug release properties of hydrogels strongly depend on its swelling characteristics [11]. The results of swelling ratios of the hydrogels synthesized with $0.25,0.5,1$ and $2 \mathrm{wt} \%$ initiator concentration (designated as I0.25, I0.50, I1.0, I2.0, respectively), 1, 2 and 3 wt\% MBA concentration (designated as MBA1, MBA2 and MBA3, respectively), 15, 20 and $25 \mathrm{wt} \%$ total monomer concentration in water (designated as TMC15, TMC,20 and TMC25, respectively), 00.0, 0.0025M, .005M,0.01M \& 0.02M Silver Nitrate (designated as SNA0, SNA1, SNA2, SNA3 and SNA4, respectively), 15, 20 and $25 \mathrm{wt} \%$ total monomer concentration in water (designated as 
TMC15, TMC,20 and TMC25, respectively) are shown in Fig.3i, ii, iii and iv, respectively. All of these swelling experiments were carried out at a $\mathrm{pH}$ of 7.6 in water.

Drug loading and entrapment efficiency of the hydrogels used for drug release study is shown in Table 1.To determine the potential application of SNA3 in drug delivery, at first we have investigated the CFx loading behavior of SNA at $30{ }^{\circ} \mathrm{C}$. The effect of the initial concentration of CFx solution on the adsorption capacities of SNA is shown in Fig.4i. The amount of the loaded drug in SNA3 was significantly affected by the impregnation times (Fig.4ii). Cumulative release profile of drug from the composite hydrogels at varied crosslinker concentration, silver nitrate concentration \& $\mathrm{pH}$ is shown in Fig. 4iii, iv, v and vi, respectively.

The initial burst release may be attributed to the release of drug associated with surface of the hydrogels. The concentration gradient of the drug between hydrogel surface and release medium (water) is the driving force for diffusion of drug from the gel network. Initial rapid release of drug is due to high concentration gradient of drug between these two phases.

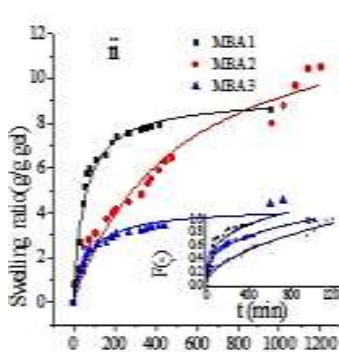

Swelling fme, $t$ (min)
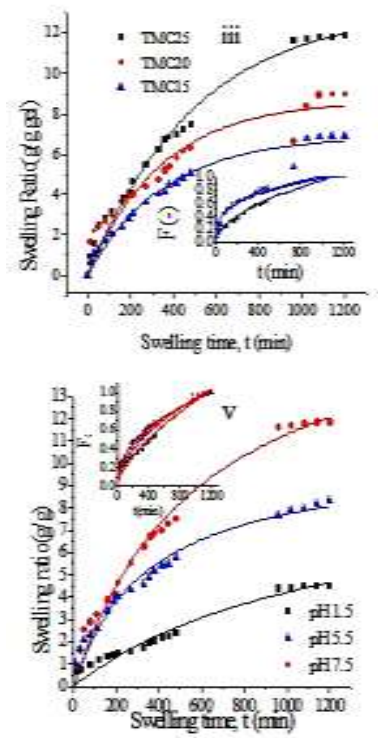

FIG.3 Effect of i) iaitiator conc., ii) crosslinker (MB.A) conc,, iii) total monomer conc., iv) silver nitrate solution conceatration and v) solution $\mathrm{pH}$, vi) Swelling-deswelling at varied

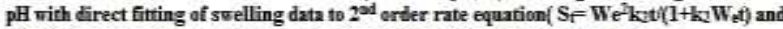
to diffusion kinetics curres (6, shorn in insets) in FG.3 i,ii,iii,iv and 8 \& $\mathrm{i}$
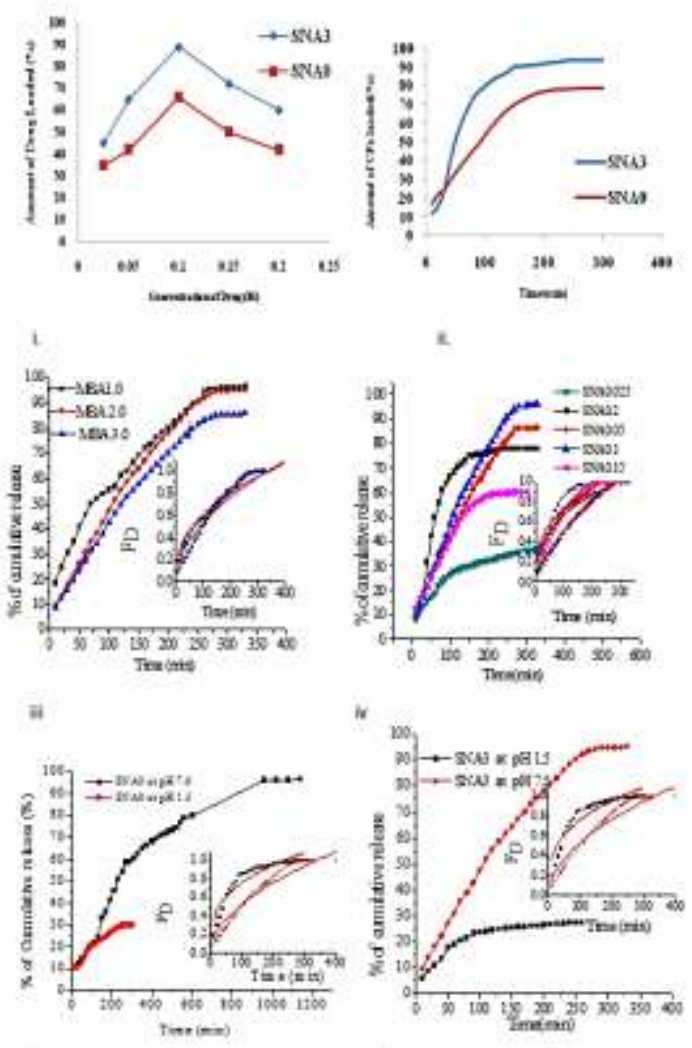

IG.4 Effect of i) Concentration depeodency of CFx loading into SNA3 and $\mathrm{SNA} A$ after $100 \mathrm{~min}$ at $30^{\circ} \mathrm{C}$. ii) Time depeadency of the drag loading amount into SNA3 and SNA0 at $30^{\circ} \mathrm{C}$ (source drug concentration=0.1 M). iii) crossliaker (MBA) conc., iv) silver aitrate conc, and v) solution $\mathrm{pH}$ on drug release from SNA0 hydrogel \& vi) solution $\mathrm{pH}$ on drug release from $\mathrm{SNA} 3$ hydrogel with direct fitting of release data to Korsmeyer-Peppas model Eq. $\mathbf{F}_{\mathbf{n}}=\mathbf{K}_{\mathbf{z}} \mathbf{t}^{\mathbf{t}}$

\section{Conclusions}

In conclusion, a facile and simple green methodology semi-interpenitrating silver nano composite hydrogels were prepared through the radical polymerization of CMC/acrylamide in water in the presence of Mango leaf 
extract,which act as a reducing agent for silver salt. The structure and swelling behaviors of the semi-IPN hydrogels were characterized by means of XRD,TEM, SEM which revealed the presence of silver nanoparticles within their networks. Modifying CMC/acrylamide and silver ion can greatly improve the swelling of corresponding silver composite hydrogel.The entrapped silver nanoparticles and ciprofloxacin molecules showed sustained release which advice enormous prolonged therapeutic values.

\section{Acknowledgements}

The authors are very much thankful to UGC-DSKothari Post Doctoral Fellowship programme for providing financial support

\section{References}

[1] K. Y. Lee, and D. J. Mooney, "Hydrogels for tissue engineering," Chemical Reviews, vol. 101, no. 7, pp. 1869-1879, 2001. https://doi.org/10.1021/cr000108x

[2] H. F. Mark, N.M.Bikals, C. G. Overberger, and J. I. Kroschwitz, Encyclopedia of Polymer Science and Engineering, Wiley-Interscience, New York, NY, USA, 1986.

[3] Mahdavinia, G. R.; Marandi, G. B.; Pourjavadi, A.; Kiani, G. "Semi-IPN carrageenan-based nanocomposite hydrogels: Synthesis and swelling behavior", Journal of Applied Polymer Science, vol.118, no. 5, pp. 2989, 2010. https://doi.org/10.1002/app.32700

[4] C. Zhou, and Q. Wu, Q, "A novel polyacrylamide nanocomposite hydrogel reinforced with natural chitosan nanofibers", Colloids and Surfaces B: Biointerfaces, Vol. 84, no. 1, pp. 155-162, 2011. https://doi.org/10.1016/j.colsurfb.2010.12.030

[5] Y. Mo, I. M"orke, and P. Wachter, "Surface enhanced Raman scattering of pyridine on silver surfaces of different roughness," Surface Science, vol. 133, no. 1, pp. L452-L458, 1983. https://doi.org/10.1016/0039-6028(83)90476-4

[6] Y.-C. Chung, I.-H. Chen, and C.-J. Chen, "The surface modification of silver nanoparticles by phosphoryl disulfides for improved biocompatibility and intracellular uptake," Biomaterials, vol. 29, pp. 1807-1816, 2008. https://doi.org/10.1016/j.biomaterials.2007.12.032

[7] N.I. Zhou, Y. Liu, L. Li, N. Meng, Y.X. Huang, J. Zhang, S.H. Wei, and S. Shen, "A new nanocomposite biomedical material of polymer/Clay-Cts-Ag nanocomposites", Current Applied Physics, vol. 7, no. 1, pp. 58-e62,2007 https://doi.org/10.1016/j.cap.2006.11.016

[8] Y.L. Luo, Q.B. Wei, F. Xu, Y.S. Chen, L.H. Fan, and C.H. Zhang, "Assembly, characterization and swelling kinetics of Ag nanoparticles in PDMAA-g-PVA hydrogel networks", Materials Chemistry and Physics, vol. 118, no. 2-3, pp. 329-336, 2009 https://doi.org/10.1016/j.matchemphys.2009.07.063

[9] Z. Jovanovi, J. Stojkovska, B. Obradovi, V. Miskovic-Stankovi, "Alginate hydrogel microbeads incorporated with Ag nanoparticles obtained by electrochemical method", Materials Chemistry and Physics, vol. 133,no. 1, pp. 182-189,2012 https://doi.org/10.1016/j.matchemphys.2012.01.005

[10] J. Xiabin, H. Yu, X. Xu, X. Chen, T. Lu, and P. Zhang, "Preparation and antibac-terial effects of PVA-PVP hydrogels containing silver nanoparticles", Journal of Applied Polymer Science, vol. 103,no. 1, pp. 125-133, 2007

https://doi.org/10.1002/app.24835

[11]Q. Wang, X.L. Xie, X.W. Zhang, J.P. Zhang, and A.Q. Wang, "Preparation and swelling properties of pH-sensitive composite hydrogel beads based on chitosan-g-poly (acrylic acid)/vermiculite and sodium alginate for diclofenac controlled release," International Journal of Biological Macromolecules, vol. 46, pp. 356-362, 2010.

https://doi.org/10.1016/j.ijbiomac.2010.01.009 